\title{
Severe insulin resistance in the setting of therapeutic hypothermia
}

Mose July, $\mathrm{MD}^{1}$, Prasanna Santhanam, $\mathrm{MD}^{1}$, Jenan Gabi, $\mathrm{MD}^{1}$, Rodhan A. Khthir, $\mathrm{MD}^{1}$

\section{Author Affiliations:}

1. Marshall University Joan C. Edwards School of Medicine, Huntington, West Virginia

The authors of this manuscript have not had a relevant duality of interest with a company whose products or services are directly related to the subject matter of their manuscript.

\section{Corresponding Author:}

Mose July, MD

Marshall University Joan C. Edwards School of Medicine

Huntington, West Virginia

Email: mosejuly@gmail.com 


\section{Abstract}

Background: Hyperglycemia is common in critically ill patients and has been associated with poor outcomes. The effect of hypothermia, whether induced or spontaneous, on insulin resistance and hyperglycemia is not well understood and sometimes overlooked.

Objectives: Goals are to review the literature on glucose metabolism and insulin resistance in patients treated with therapeutic hypothermia $(\mathrm{TH})$ after sudden cardiac arrest (SCA) and also to present knowledge about the possible effects of hypothermia on glycemic control and insulin sensitivity.

Methods: We present an example of extreme insulin resistance during the period of therapeutic hypothermia in a patient who was admitted after a sudden cardiac Arrest. Literature review was conducted as a Medline/PubMed search. Studies were assessed regarding designs, primary and secondary efficacy parameters on TH after sudden cardiac arrest and effects on glucose metabolism.

Results: A 68-year-old male who was admitted after SCA was noted to have extreme insulin resistance during $\mathrm{TH}$ which completely resolved during the rewarming phase of $\mathrm{TH}$. No randomized, controlled trials were found in the Medline search. Two studies found that there was an increase in insulin resistance during TH especially during the cooling phase, whereas three studies found no direct association between $\mathrm{TH}$ and blood glucose levels.

Conclusion: There is a possible association between $\mathrm{TH}$ and a reversible form of insulin resistance. More studies are needed. Patients who are treated with TH after SCA may present a challenge in glucose management.

\section{Keywords}

therapeutic hypothermia, insulin resistance, sudden cardiac arrest

\section{Introduction}

Therapeutic hypothermia (TH) has resulted in improvements in mortality rates from sudden cardiac death. ${ }^{1}$ Literature on the effect of hypothermia on insulin resistance and hyperglycemia is lacking. We describe a patient who developed transient severe insulin resistance during the induced hypothermia phase with clear temporal association between low body temperature and high insulin requirement. We also review the current literature with respect to glucose metabolism in post cardiac arrest patients undergoing $\mathrm{TH}$.

\section{Case Report}

A 68-year old Caucasian man with type 2 diabetes mellitus presented after an out of the hospital sudden cardiac arrest (SCA). He was admitted after the return of spontaneous circulation (ROSC) with defibrillator shock and Amiodarone $150 \mathrm{mg}$ administered intravenously for ventricular fibrillation. Upon arrival to the emergency room, he was found to have ST-elevation 
myocardial infarction (STEMI) and was sent to the cardiac catheterization lab where no culprit lesion was identified. On initial neurological examination, he had minimal response to deep pain stimuli, inability to follow instructions, no purposeful movements with normal deep tendon reflexes and muscular tone. Therapeutic hypothermia was initiated and monitored in the intensive care unit. He also received renal replacement therapy for acute renal injury presumably due to severe hypoxic end-organ damage. His body mass index was $34 \mathrm{~kg} / \mathrm{m}^{2}$ with no signs of Cushing's syndrome or Acromegaly.

His past medical history was significant for hypertension, hyperlipidemia and coronary artery disease (he had an angioplasty done 3 years prior to presentation). His home regimen for diabetes consisted of metformin and NPH insulin (doses were unclear). Initial blood glucose (BG) was $238 \mathrm{mg} / \mathrm{dl}$ in the Emergency room. After initiation of therapeutic hypothermia he developed severe hyperglycemia while on continuous insulin infusion (CII) protocol, reaching a maximum of $434 \mathrm{mg} / \mathrm{dl}$ within the first 24 hours of TH. The hourly intravenous insulin infusion rate increased to a maximum of 280 units/hour (Figure 1). Eventually his blood glucose reached target of 140-180 mg/dl during the rewarming phase as his insulin requirements and blood glucose levels started to decrease. Blood glucose level remained between 80 and $100 \mathrm{mg} / \mathrm{dl}$ without CII after the rewarming phase. He was not receiving enteral or parenteral nutrition. Baseline laboratory values showed renal impairment with metabolic acidosis. Liver function test was normal (Table 1).

Table 1. Laboratory results at the time of admission

\begin{tabular}{|l|l|}
\hline LAB & $\begin{array}{l}\text { VALUE } \\
\text { (reference range) }\end{array}$ \\
\hline Sodium $(\mathrm{mEq} / \mathrm{L})$ & $132(135-145)$ \\
\hline Potassium $(\mathrm{mEq} / \mathrm{L})$ & $3.8(3.3-5.1)$ \\
\hline Chloride $(\mathrm{mmol} / \mathrm{L})$ & $100(96-108)$ \\
\hline CO2 $(\mathrm{mmol} / \mathrm{L})$ & $18(21-32)$ \\
\hline BUN $(\mathrm{mg} / \mathrm{dL})$ & $43(7-18)$ \\
\hline Creatinine (mg/dL) & $2.3(0.6-1.1)$ \\
\hline Calcium $(\mathrm{mg} / \mathrm{dL})$ & $6.8(8.5-10.1)$ \\
\hline Anion Gap (mmol/L) & $13(4-16)$ \\
\hline Albumin (g/dL) & $1.9(2.9-4.9)$ \\
\hline
\end{tabular}

\begin{tabular}{|l|l|}
\hline LAB & $\begin{array}{l}\text { VALUE } \\
\text { (reference range) }\end{array}$ \\
\hline Total Bilirubin $(\mathrm{mg} / \mathrm{dL})$ & $0.5(0.1-1.0)$ \\
\hline AST (U/L) & $87(5-37)$ \\
\hline ALT (U/L) & $56(15-65)$ \\
\hline $\begin{array}{l}\text { Alkaline phosphatase } \\
(\mathrm{U} / \mathrm{L})\end{array}$ & $49(50-120)$ \\
\hline pH & $7.20(7.35-7.45)$ \\
\hline Hemoglobin $(\mathrm{g} / \mathrm{dL})$ & $11.7(12.0-15.0)$ \\
\hline Lactate $(\mathrm{mmol} / \mathrm{L})$ & $1.9(0.4-2.0)$ \\
\hline Hemoglobin A1c $(\%)$ & $7.7(4.0-6.0)$ \\
\hline WBC $\left(x 1000 / \mathrm{mm}^{3}\right)$ & $8.7(4.8-10.8)$ \\
\hline
\end{tabular}


Figure 1. Blood glucose levels and insulin infusion rates during therapeutic hypothermia. Adm = admission; $\mathrm{TH}=$ onset of therapeutic hypothermia

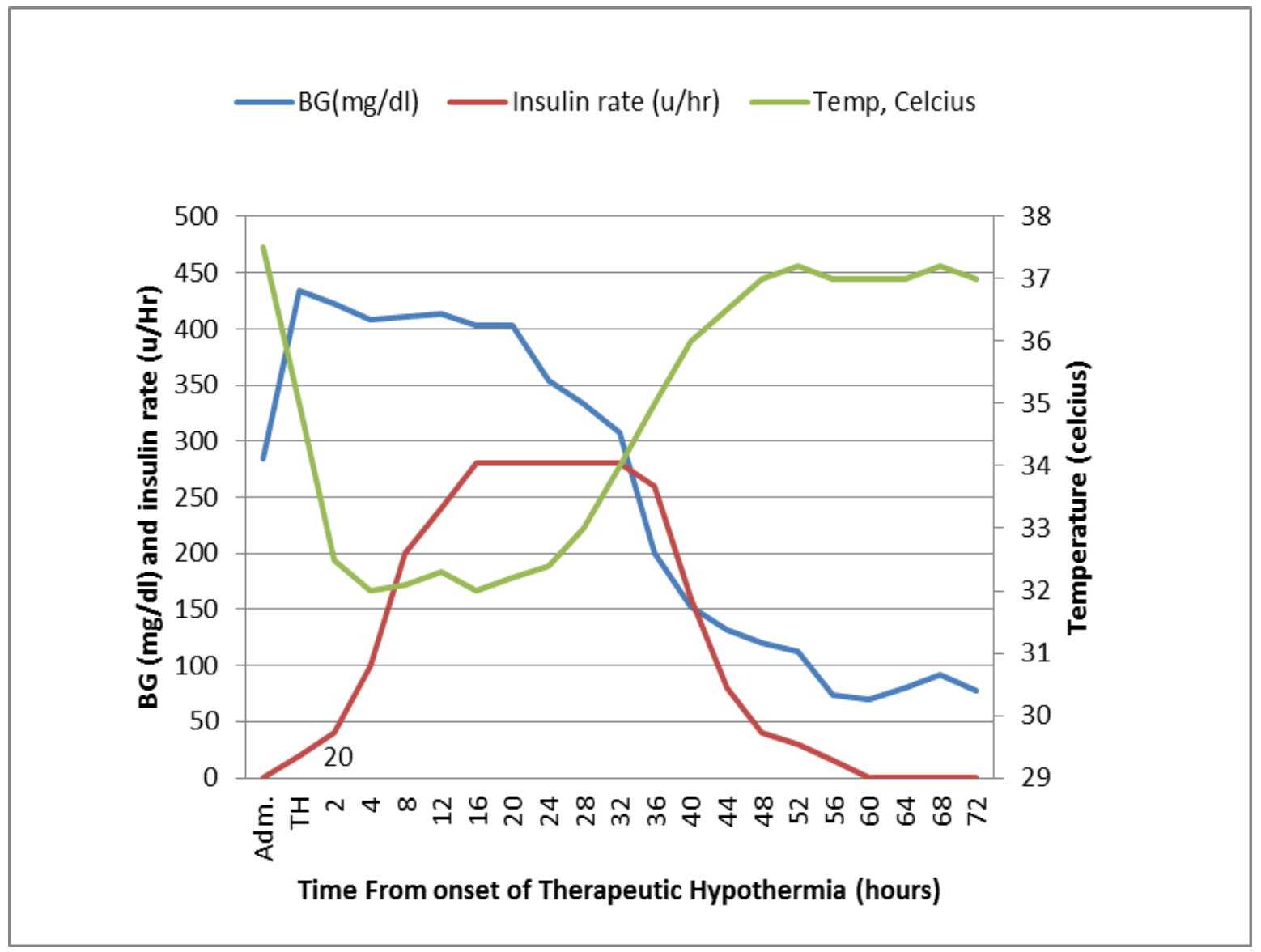

The insulin autoantibodies were negative and insulin level during the plateau phase of hypothermia was extremely high (>1000 Miu/ml), indicating extreme insulin resistance. There was no evidence of any other medical intervention or condition that might have confounded our results (like sepsis or administration of glucocorticoids). After discontinuation of therapeutic hypothermia, his insulin requirements and his blood glucose decreased rapidly as illustrated in figure 1. After that he did not require any insulin to keep his blood glucose at target for the last 5 days of his hospitalization before he died of anoxic brain damage.

\section{Discussion}

Our patient demonstrated evidence of extreme insulin resistance in the setting of therapeutic hypothermia, followed by a marked improvement with the return of normal body temperature. There was a clear temporal association between body temperature and insulin requirement which may suggest that hypothermia might have contributed to the extreme insulin resistance in our patient. 
Hyperglycemia is common after CA and is associated with unfavorable neurologic outcomes and mortality. ${ }^{2-4}$ Insulin resistance (IR) has also been observed in cardiac arrest patients treated with $\mathrm{TH}^{5-7}$

In an observational study involving 103 patients who underwent TH for SCA, Kim et al showed that early achievement of target BG level was significantly associated with better survival and favorable neurologic outcomes. ${ }^{8}$ In this study, those patients who reached the target range within 4 hours were more likely to survive and to be discharged with favorable neurologic outcomes, regardless of other confounding factors such as pre-arrest variables, insulin requirements, and previous history of diabetes. The study did not show a clear association between $\mathrm{TH}$ and insulin resistance.

Ettleson et al conducted a retrospective chart review of 62 post-cardiac arrest patients undergoing TH in a tertiary institution between September 2005 and April 2008. ${ }^{9}$ In their study they found increased mortality associated with increased blood glucose levels after SCA but before initiation of TH or an insulin drip. TH had no independent effect on BG.

Other studies did show an association between TH and insulin resistance. In a study of 200 post cardiac arrest patients, Pri and coworkers found a significant increase in insulin sensitivity and decrease in insulin-sensitivity variability as persons transitioned from the cool to warm phase of TH. ${ }^{10}$ The study by Cueni-Villoz et al showed that besides acute stress from cardiac arrest, therapeutic hypothermia has a role in causing hyperglycemia and high glucose variability through a reversible increase in insulin resistance during the cooling phase. ${ }^{11}$

Forni et al showed in a retrospective study that there was a significant increase in mean blood glucose in the cooling phase as compared to rewarming $(9.16 \pm 3.22 \mathrm{mmol} / \mathrm{L}$ vs. $6.54 \pm 2.45$ $\mathrm{mmol} / \mathrm{L} ; \mathrm{P}<0.01)$ with higher insulin requirements $(15.2 \pm 16.1$ vs. $7 \pm 12.5$ units $/ \mathrm{h}) .{ }^{12}$

\section{Conclusion}

We presented a patient with extreme insulin resistance that developed during the cooling phase of therapeutic hypothermia and resolved completely during the rewarming phase. In our patient we reduced and subsequently stopped insulin infusion during the warming phase in a rapid way (from > 250 units per hour to zero) to avoid hypoglycemia which can be detrimental in a patient with anoxic brain injury (Figure 1). Review of the literature on this topic showed conflicting data regarding $\mathrm{TH}$ as an independent risk factor for hyperglycemia and insulin resistance. It is possible that certain individuals are more prone to the metabolic effects of hypothermia. Further studies are needed to confirm the association and to identify the predictors for such severe form of insulin resistance so that a specialized insulin infusion protocol can be used. Physicians and nurses need to be aware of this potential association and the potential need for a modified intravenous insulin protocol for patients who develop severe insulin resistance during the cooling phase of TH. 


\section{References}

1. Bernard SA, Gray TW, Buist MD, Jones BM, Silvester W, Gutteridge G, et al. Treatment of comatose survivors of out-of-hospital cardiac arrest with induced hypothermia. The New England journal of medicine. 2002;346(8):557-63.

2. D.G. Beiser, G.E. Carr, D.P. Edelson, M.A. Peberdy, T.L. Hoek. Derangements in blood glucose following initial resuscitation from in-hospital cardiac arrest: a report from the national registry of cardiopulmonary resuscitation. Resuscitation, 2009;(80):624-630.

3. S.H. Kim, S.P. Choi, K.N. Park, S.J. Lee, K.W. Lee, T.O. Jeong, et al. Association of blood glucose at admission with outcomes in patients treated with therapeutic hypothermia after cardiac arrest. Am J Emerg Med. 2014;(32): 900-904.

4. N. Nielsen, K. Sunde, J. Hovdenes, R.R. Riker, S. Rubertsson, P. Stammet, et al. Adverse events and their relation to mortality in out-of-hospital cardiac arrest patients treated with therapeutic hypothermia. Crit Care Med. 2011; (39):57-64.

5. Nurmi J, Boyd J, Anttalainen N, Westerbacka J, Kuisma M. Early increase in blood glucose in patients resuscitated from out-of-hospital ventricular fibrillation predicts poor outcome. Diabetes Care. 2012;(35):510-512.

6. Polderman K. Application of therapeutic hypothermia in the intensive care unit. Intens Care Med. 2004;30:757-769.

7. Busch M, Soreide E, Lossius HM, Lexow K, Dickstein K. Rapid implementation of therapeutic hypothermia in comatose out-of hospital cardiac arrest survivors. Acta Anaesthesiol Scand. 2006; 50:12771283.

8. Kim et al. Time to reach target glucose level and outcome after cardiac arrest patients treated with $\mathrm{t}$ herapeutic hypothermia. J Cri Care. 2015;Dec;30 (6) 1204-9.

9. Ettleson MD, Arguello V, Wallia A, Arguelles L, Bernstein RA, Molitch ME. Hyperglycemia and insulin resistance in cardiac arrest patients treated with moderate hypothermia. J. Clin. Endocrinol. Metab. 2014;99(10):E2010-4.

10. Sah Pri A, Chase JG, Pretty CG, Shaw GM, Preiser JC, Vincent JL, et al. Evolution of insulin sensitivity and its variability in out-of-hospital cardiac arrest (OHCA) patients treated with hypothermia. Critical care. 2014;18(5):586.

11. Cueni-Villoz N, Devigili A, Delodder F, Cianferoni S, Feihl F, Rossetti AO, et al. Increased blood glucose variability during therapeutic hypothermia and outcome after cardiac arrest. Critical care medicine. 2011;39(10):2225-31.

12. Forni AA, Rocchio MA, Szumita PM, Anger KE, Avery KR, Scirica BM. Evaluation of glucose management during therapeutic hypothermia at a Tertiary Academic Medical Center. Resuscitation. 2015 Apr;89:64-9. 